\title{
Réanimer le musée. Récit et analyse d'une expérience de recherche-création
}

\section{Shirley Niclais}

«Vivre notre musée » est le titre d'une résidence-mission que j'ai menée entre juin 2017 et juin 2018 dans un musée d'art et d'histoire de région parisienne. À l'origine, je proposais plutôt l'idée d'« Habiter le musée ». Jugée trop intrusive, ma proposition a donc été aménagée, repensée par mon équipe encadrante. Entre vivre et habiter, quelles différences ? Que doit-on y comprendre ? J'ai accepté « vivre » pour sa résonance avec l'idée d'y introduire les arts du spectacle vivant, mais force est de constater que j'ai bien « habité » le musée et l'ai effectivement fait vivre, autrement.

Durant cette année expérimentale, en plasticienne-performeuse, metteure en scène, marionnettiste et chercheuse, j'ai souhaité transformer le lieu patrimonial en un lieu vivant, vibrant des présences passées et présentes. À travers des ateliers de recherche-création avec des publics variés (enfants, adolescents, personnes en situation de handicap ou de détresse sociale, séniors et même avec l'équipe des agents du musée), il s'est agi de se saisir des collections d'objets, d'archives, et des œuvres d'art exposées pour en faire le matériau d'un nouveau théâtre. Il s'est agi de jouer, de rejouer et de déjouer le musée pour mieux le réanimer.

Avec pour point d'ancrage le sous-projet phare Les habitants du seuil, diptyque film et pièce vivante pour marionnettes, créé d'après Joseph Beuys et Franz Kafka au cours de cette résidence, je me propose, près d'un an et demi après l'avoir achevée, de porter un regard critique et analytique sur cette expérience. En témoignant de ce parcours, il faut aussi se confronter à ses écueils. Mon présupposé théorique issu de mes recherches doctorales sur la théâtralité du muséal s'est en effet heurté à la réalité du terrain. Comment faire théâtre $d u$ musée ou au musée ? Et quelles différences cela fait-il ?

Cet article-témoignage retrace ce parcours singulier. Privilégiant la forme d'un va-etvient, aller-retour entre les perspectives et les contraintes, les préconçus et les réalisations concrètes, ce texte invite à un dialogue entre la recherche et la création autant qu'entre l'institution et l'artiste. Il s'organise en trois temps presque chronologiques : les désirs de l'avant-projet (le musée ou le temps suspendu), les questionnements du pendant (un territoire à habiter) et l'expression du mouvement d'une recherche en actes, toujours à l'œuvre (rematérialiser la rencontre). 


\section{Le musée ou le temps suspendu}

Voici donc mon idée de départ, comme un fil rouge : celle d'un « ci-gît », d'un temps suspendu mais en devenir, en construction ou en vivance, c'est-à-dire en mouvement permanent. J'avais en tête, en pensant à construire un projet dans un petit musée municipal, une forme de cristallisation du temps. La conservation est-elle une pétrification ? Le musée n'est-il condamné qu'à exposer un ce-qui-a-été mais qui n'est plus ? Peut-il encore se penser et se vivre au présent, vibrer, exister ? Et comment ? Je pensais beaucoup à Antonin Artaud et à son refus du chef-d'œuvre. J'avais en tête, éparses et sans doute défaites par le souvenir flou que j'avais alors de leur lecture, ses diatribes spectaculaires, disant qu'il fallait brûler la bibliothèque d'Alexandrie, que toute archive était vaine, qu'il fallait en finir avec les chefsd'œuvre. Ce que vous appelez mes œuvres ne sont que des déchets de moi-même, écrivait-il si bien. J'avais pourtant, et parallèlement, une autre idée du musée : celle d'un espace-temps où tout est suspendu, rien ne meurt, tout est éternel, conservé, préservé. Le musée, cet espacepoème où tout se chuchote, est le lieu même de la contemplation d'un passé qu'il est possible de réactualiser. Ici, la visite est gratuite, les portes ouvertes, la lumière est tamisée, et les espaces - bien que restreints - sont presque toujours vides. Certains le disent « charmant», d'autres «vieillot». Le musée municipal est un abri, une coquille, un terrier, dans lequel on pourrait venir se blottir pour se souvenir.

Nous ne parlons pas ici d'un musée qui se consomme, où se déversent des dizaines de milliers de touristes. Je percevais le musée municipal comme un lieu qui échappe aux injonctions contemporaines : production, dévoration. Bien sûr, j’avais lu Michel Foucault et ses mots résonnaient encore dans ma tête au moment d'écrire mon dossier de candidature :

Les musées, comme les bibliothèques sont des hétérotopies du temps qui s'accumule à l'infini : l'idée de tout accumuler, d'arrêter le temps ou plutôt de le laisser se déposer à l'infini dans un certain espace privilégié, l'idée de constituer l'archive générale d'une culture, la volonté d'enfermer dans un lieu tous les temps, comme si cet espace pouvait être définitivement hors du temps, c'est là une idée tout à fait moderne : le musée et la bibliothèque sont des hétérotopies propres à notre culture. (30)

Le musée municipal n'abrite pas seulement des œuvres, il se fait l'écrin des traces de relations passées, présentes et à venir : entre l'artiste et son œuvre, entre le regardeur et l'objet exposé, entre un regardeur et un autre regardeur, dans le partage des gestes de spectation. Au centre, il y a la ville, médiatrice de toutes ces rencontres. Comme elle, le musée évolue, se transforme : territoire en mutation permanente. C'était là justement le thème décrit par l'appel à projet auquel j'ai répondu : «territoire en mutation ». 


\section{Gestes artistiques}

Aux yeux du jury d'attribution des bourses de résidence, ma posture était plutôt originale et mon parcours qualifié d'assez éclectique. J'ai une double, voire triple formation : je suis marionnettiste, actrice corporelle et artiste de performance. Parallèlement à ma formation artistique, je terminais une thèse de doctorat qui invitait à penser ensemble les arts du spectacle et les arts plastiques, deux disciplines que j'enseignais alors et enseigne toujours à l'université. Je me suis présentée comme praticienne de «recherche-création » et ma candidature a donc été retenue. J'ai décroché le Contrat local d'éducation artistique (CLEA) associé à cet appel à projets.

La résidence, qui n'avait pas vocation à être de création, et encore moins de recherche (au sens académique du terme) était d'un genre bien particulier. Il s'agissait d'une résidencemission. Les artistes engagés dans ce genre de dispositifs, a priori largement soutenu sur le plan politique puisqu'elles dépendent de subventions exclusivement publiques, sont invités à y produire des «gestes artistiques » en collaboration avec un public le plus mixte, nombreux et varié possible. Par ces gestes artistiques, il faut toucher la population locale, lui faire rencontrer l'art et les artistes.

François Annycke, missionné par la DRAC (Direction régionale des affaires culturelles) de la région Nord-Pas-de-Calais pour rendre compte des «gestes artistiques » réalisés dans le cadre des CLEA de sa région est l'auteur d'un texte dont l'un des objectifs est d'expliciter ce dont il s'agit. En exergue de son texte, c'est à la définition de Giorgio Agamben qu'il invite à se référer :

Geste est le nom de cette croisée où se rencontrent la vie et l'art, l'acte et la puissance, le général et le particulier, le texte et l'exécution. Fragment de vie soustrait au contexte de la biographie individuelle et fragment soustrait au contexte de la neutralité esthétique : pure praxis. Ni valeur d'usage, ni valeur d'échange, ni expérience biographique, ni événement impersonnel, le geste est l'envers de la marchandise. (90) 


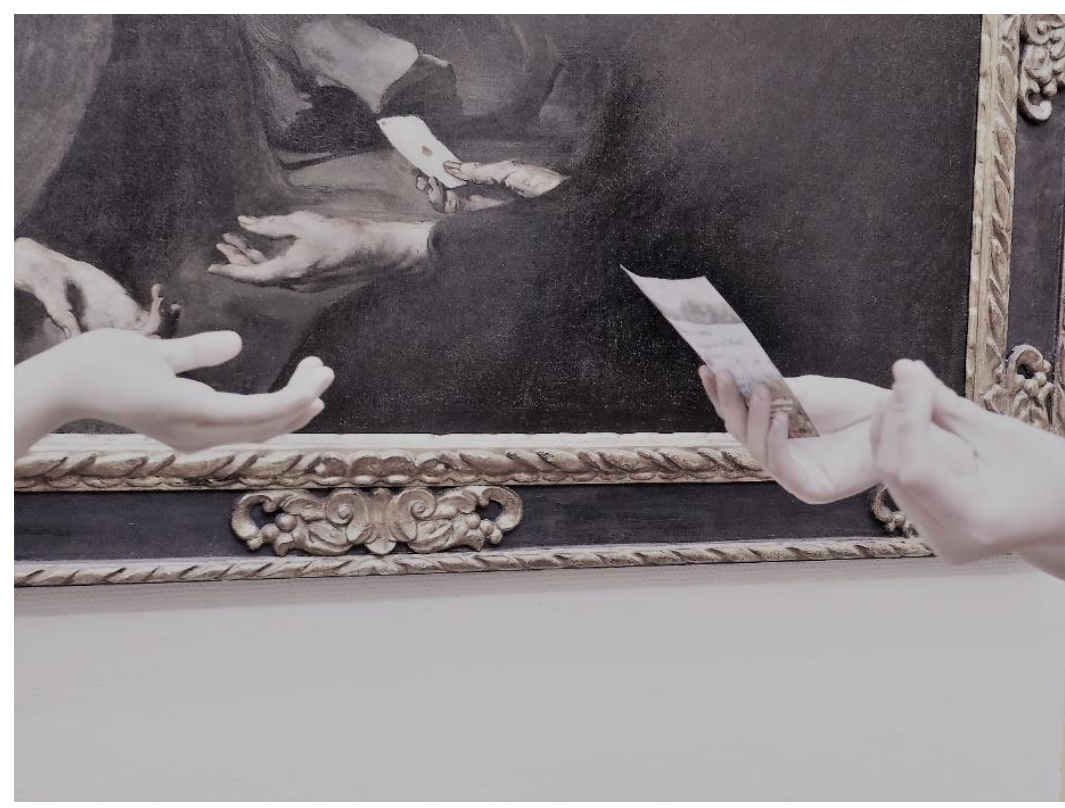

Fig. 1. «Vivre notre musée », recherche de médiation alternative avec de jeunes adultes en situation de handicap (Shirley Niclais, 2018)

Annycke précise que le geste est un mouvement du corps révélant un état d'esprit ou visant à exprimer, à exécuter quelque chose. L'action dont il est question ici mobilise le corps, l'esprit, a une signification, un sens, un but qualifié d'artistique, c'est-à-dire une activité créatrice, esthétique, la production de quelque chose d'original, de créatif, explique-t-il encore. Ce n'est pas une création, mais le partage, à plus ou moins grande échelle, d'un processus de création, un dialogue qui passe par l'art, la pratique, cette « praxis » évoquée par Agamben. Il ne faut pas créer d'œuvre d'art mais poser la question, par le geste, de la possibilité de son émergence. Pour moi, il s'agissait donc bien d'une recherche, d'un travail de fouille, résolument expérimentale qui, partagée avec des publics variés, devait donner lieu à des éléments de «cristallisations visibles » marquant les différentes étapes de cette recherche en acte(s). Pour moi, il s'agissait là d'un terrain parfait pour faire l'expérience, le terrain, de ce qui animait déjà ma recherche personnelle, qu'elle soit académique ou artistique.

\section{La recherche-création}

Depuis 2011, je pense et conçois des performances, des écritures de plateau et des installations à activer par le corps, la voix et l'objet qui sont au cœur des dispositifs que je mets en place. Ce sont mes médiums principaux. Le sens premier de mon travail est la collaboration et la synergie (avec d'autres artistes ou des professionnels dont je peux réinvestir la spécialité : des plasticiens, des musiciens, des techniciens) ou avec un certain type de public : bien sûr des étudiants à l'université (c'est le public que je connais le mieux), mais aussi les collégiens et 
plus récemment des adultes en situation d'exclusion sociale. Je pratique presque exclusivement l'in-situ. L'idée pour moi est d'habiter un espace, investir un territoire, pour le voir réagir à une présence, et moi-même m'adapter à de nouvelles données, de nouveaux rythmes. La posture qui m'intéresse c'est celle d'un virus qui tout à la fois prend de son environnement et y apporte quelque chose. Ma formation de marionnettiste m'invite à toujours partir d'un socle de réalité qui peut prendre la forme de document, d'archive, évidemment d'objet comme arraché au réel ou à la matière. Dans le cadre de mon travail, je rencontre donc des œuvres. Qu'elles soient des objets physiques, des textes ou des expériences, je les réinvestis et les poétise, pour mieux les questionner. Les objets des autres font donc partie de mon parcours, c'est cela qui explique mon intérêt spécifique pour le musée, ce lieu où précisément l'on rassemble les objets des autres, où cohabitent l'actuel et le passé. Comme enseignante en Histoire de l'art à l'université, j'ai questionné le rapport physique et sensible que l'on entretient avec les œuvres dans un espace muséal. Qu'est-ce que « rencontrer une œuvre»? Qu'est-ce qu'elle nous fait dire ? Qu'est-ce qu'elle nous fait (res)sentir ? Comment elle nous bouge ? Comment elle nous danse ? Dans le cadre de l'écriture de ma thèse de doctorat, j'ai notamment défendu l'idée selon laquelle l'espace muséal est un terrain propice à l'émergence du spectacle vivant. Les objets muséaux eux-mêmes peuvent ouvrir des espaces théâtraux, théâtralisés et théâtralisables.

C'est de cette manière que je voulais vraiment habiter le musée. Je voulais l'occuper, ne pas le quitter, le faire vivre de bout en bout, jour après jour, y organiser des performances in situ, éventuellement des conférences-performances plus explicitement liées à mon travail académique, y engager des actions participatives autour des collections, des évènements décalés. Le projet que j'ai présenté au jury de sélection pour cette résidence-mission consistait donc en la réalisation de modules performés par et pour les publics du musée. J'avais pour objectif de documenter chacun de ces modules (par l'image, le texte, les enregistrements audio ou vidéo) de telle sorte qu'une archive vivante puisse être conservée à l'issue de la résidence et constituer une matière pérenne pouvant effectivement faire l'objet d'une exposition générale de restitution. Mon idée d'origine pour ce projet émergeait d'une réflexion très pragmatique : qu'est-ce qu'un musée municipal ? Qu'est-ce qu'on n'y fait? Qu'est-ce qu'on y expose ? Qui le visite et pourquoi ? Le musée est-il bien un reflet en miroir du territoire en mutation sur lequel il s'inscrit? Ce reflet est projeté dans l'adéquation ou l'inadéquation des corps qui le visitent, des personnalités qui l'animent et des objets patrimoniaux qui y sont conservés. Le musée municipal, c'est peut-être plutôt l'écrin (physique) de l'identité mouvante d'un territoire. Ma question, très simple, était donc la suivante : comment s'y reconnaitre encore aujourd'hui ? Comment le public peut-il investir cette mémoire ? De quels passages le musée est-il la trace ? 
Si je suis l'habitant d'une ville, le musée municipal doit-il vraiment me ressembler ? Et si oui, comment? Qu'est-ce qui résonne en moi et participe à constituer mon expérience de la ville comme mon identité d'habitant?

Le travail à engager oscillait donc entre l'image fixe de la collection (comme collection) et le mouvement de ces habitants. Le territoire est en mutation parce que ses habitants sont en mutation, parce que son identité est mouvante. Il pouvait donc s'agir de s'approprier ce patrimoine en le performant pour s'offrir la possibilité de porter ce masque et de l'interroger. Le temps de la performance aurait pu alors réunir en un même espace les différentes temporalités de la ville, sa richesse culturelle d'hier et celle d'aujourd'hui, son patrimoine et son actualité à travers la superposition des visages qui l'incarnent avec ceux qui l'ont incarnée.

\section{Un territoire à habiter (et à questionner)}

Je ne suis pas la première à me poser ce genre de question. Depuis 2012, par exemple, le groupe de recherche « la performance théâtrale au musée ${ }^{1}$ », devenu «Créons au musée » dirigé par Katia Légeret ${ }^{2}$, propose des performances inter-artistiques collectives, s'interrogeant toujours sur les nouvelles postures et gestes que peuvent prendre les visiteurs des espaces muséaux, aujourd'hui, au vingt-et-unième siècle. Dans son « introduction à la performance des arts vivants au musée : recherches-créations »(9-65), Légeret, rappelle elle-même combien l'actualité du monde muséal est ouverte à ce genre de proposition. Elle cite notamment le rapport de la commission «Musées XXIème siècle » du Ministère de la Culture et de la Communication, rédigé sous la direction de Jacqueline Eidelman, qui souligne la fonction de plus en plus importante du musée en tant que lieu de vie, et l'importance d'une participation conjointe d'artistes du spectacle vivant et de visiteurs. Il s'agit là de penser l'avenir d'une pratique : celle d'être visiteur d'un lieu muséal. Sur cette question, elle renvoie également à la lecture de ce texte phare de la muséologie actuelle et contemporaine, celui de la conservatrice en chef du Patrimoine Catherine Grenier, La fin des musées? Dans ce texte, l'actuelle directrice de la fondation Giacometti et ancienne directrice adjointe du Musée National d'Art Moderne s’interroge sur la nécessité de faire évoluer les modèles muséaux et insiste sur la nécessité d'en construire de nouveaux en étroite collaboration avec les artistes et les chercheurs - dont les mondes cohabitent déjà si difficilement en France. Elle s'interroge sur la place du musée de demain au regard des bouleversements économiques, de l'envolée du marché de l'art, des évolutions démographiques et du développement de la communication globale et des outils numériques. La mutation des publics et les transformations des pratiques culturelles sont au 
cœur de son ouvrage, de son constat initial (porté par la question du titre) et de ses propositions. Au-delà de son fonctionnement, c'est l'identité du musée qu'elle décrit comme « en crise ».

Grenier en propose un nouveau modèle, polymorphe, qui transcende la séparation entre musée d'art et de civilisation, et s'incarne en une série de formules-idées autour desquelles s'articule son ouvrage : «musée dynamique », «musée monde », « musée cité », «musée campus », « musée public », « musée forum ». Elle termine son ouvrage par un dernier chapitre intitulé «le musée et les artistes ». Pour les créateurs contemporains, y explique-t-elle, «le musée n'est pas seulement le lieu de la reconnaissance. Il est tout d'abord le lieu d'initiation et de formation, puis ensuite un espace de propositions intellectuelles et de suggestions » (129). Pourtant,

Les musées ont la tâche délicate de gérer des exigences contradictoires. S'ils se sont progressivement adaptés aux impératifs de formes artistiques de plus en plus complexes à mettre en œuvre, les contraintes liées à l'accueil d'un public toujours plus nombreux, celles de la mise en sécurité des visiteurs et des œuvres, celles de la conservation préventive, viennent souvent contredire ou amoindrir l'exécution intégrale des dispositions conçues par les artistes. Entre la préservation de la volonté de l'artiste et les mesures de conservation de l'œuvre, l'écart s'est creusé, du fait, notamment, des nouveaux matériaux techniques utilisés par les créateurs et de l'augmentation considérable de la fréquentation. (130)

Si, comme je l'ai déjà écrit, le musée dans lequel je suis intervenue entre 2017 et 2018 n'a pas à souffrir d'une augmentation considérable de la fréquentation qui met en péril la sécurité des œuvres et des visiteurs, il est, à son échelle, confronté aux mêmes enjeux. Un musée municipal comme celui-là doit composer avec, d'une part, l'envie sincère de se moderniser, d'accueillir la création contemporaine qui, peut-être, pourrait lui permettre de conquérir de nouveaux publics, d'actualiser ses collections et, d'autre part, une mission comme chevillée au corps des dirigeants de conserver, de protéger, jusqu'à parfois ne plus montrer ce que le temps a rendu trop fragile. Le temps passé aux acquisitions et aux préservations du patrimoine excède alors celui qui permettrait les rencontres, les médiations, les accompagnements, et les prises de risque, aussi.

\section{Les éclats du projet}

Dans la cadre de ma résidence, j'ai donc été amenée à proposer une série d'ateliers de création à des habitants des deux villes partenaires du projet. À partir d'expérimentations sensibles autour des collections, j'ai proposé aux participants de devenir des témoins-acteurs des mutations du territoire de la ville en s'appropriant les objets exposés. Entre arts plastiques, 
théâtre et mouvement, chacun a été invité à créer avec moi des dispositifs (installations, performances, dessins et vidéos) à activer par la présence, la voix et le geste à partir des sensations ou souvenirs évoqués par l'espace muséal et ce qu'il abrite. Pendant ma résidence, les œuvres et les objets du musée ont été regardés, commentés, bougés, transformés. Ce qui s'est produit n'aura été que la cristallisation de la vie du musée, et les «gestes artistiques » présentés auront permis de faire éclater pour mieux les révéler ses principes fondateurs. Comme l'indique le titre donné à l'évènement de restitution finale, présentée sous la forme d'une exposition dansée, depuis le musée et vers la ville, en extérieur, le musée est entré en résonnance. Il s'agissait, une dernière fois, de déployer et de déplacer hors du musée, directement sur le territoire, ces matériaux physiques, gestuels, plastiques - pour ne pas dire performatifs - qui nous avaient tous occupés durant des mois. Il m'a semblé que le lien avec l'espace du musée pouvait inviter à des expérimentations fécondes autour de cette thématique de la mutation d'un territoire : de ce qui traverse et laisse des traces qui peuvent s'imprimer dans les murs et hors les murs du musée ; de faire jouer la ville et la collection de la ville, en miroir. Il s'agissait de développer un dernier travail sur les réalités et les virtualités de présence du musée dans la ville - de jouer sur les effets de présence, de les stimuler - de confronter les cartels à leur réalité dans la ville : images exposées dans l'espace public, distribuées dans la ville par les jeunes performeurs impliqués lors de cette dernière étape, vidéos projetées hors du musée. Il fallait tirer une dernière fois les fils d'un ensemble éclaté, fragmenté. Il fallait tisser l'ensemble, conclure et rappeler tout ce que nous avions traversé.

D'abord, il y a eu ces expériences de médiation sensible avec les publics - entre pratique et théorie. J'avais imaginé des séances d'ateliers hybrides entre recherches sur le patrimoine du musée, et investissement dans une pratique corporelle et vocale autour de la question : comment rencontrer une œuvre ? Comment s'y connaitre ou comment s'approprier une œuvre, un patrimoine? Ensuite, ces cartographie(s) poétique(s) qui permettaient d'adopter un nouveau regard sur les trajectoires quotidiennes dans l'espace du musée (en se saisissant du concret des déplacements) pour commencer à déréaliser et poétiser l'espace. Lors d'ateliers avec différents acteurs locaux (équipe du musée, autant que différents types de publics en visites), ces trajectoires avaient varié. Comment les réunir ? Bien sûr, les performance( $s$ ) dont découlaient ces trajectoires identifiées. J'ai proposé aux participants d'imaginer avec moi des modules de médiations performées dans les espaces du musée à partir de ces déplacements. Enfin, l'archivage que j'ai voulu vivant. Tout au long de ces différentes étapes, il s'agissait d'archiver les traces des actions menées. 
Il ne peut en effet jamais s'agir tout à fait d'une pratique purement et simplement expérientielle, vivante et éphémère. Il faut rendre des comptes, justifier, (re)présenter à ceux qui n'étaient pas là, expliquer comment pourquoi et pour quels résultats. Il faut rationnaliser l'ir-rationnalisable. Il faut donc que le vivant s'organise pour s'exposer, qu'il se conserve encore, se calcifie toujours pour s'archiver de nouveau. Cette perspective, que j'avais pourtant anticipée, est devenue dévorante. Mon appareil photo sans cesse à la main, il fallait que je sois là et déjà ailleurs, en acte, en geste, en partage et déjà dans une logique de conservation : prise à mon propre jeu. Photographie, film, texte, réunion, reformulation, re-questionnement, réadaptation. Une grande partie du travail s'est révélée être un geste de communication.

De cette sincère volonté d'ouverture à des pratiques contemporaines, il fallait d'abord longuement parler avant de pouvoir enfin en expérimenter les enjeux. Il fallait toujours, avant même de créer, être en mesure de défendre le bilan, tant sur le plan artistique que politique, social et économique. C'est bien de l'image de la ville dont il s'agissait, et alors la possibilité d'une censure n'est jamais loin. Il faut correspondre à ce qui est attendu, à des logiques qui m'ont d'abord échappées.

\section{Confronter l'écrin au terrain}

Je pensais n'avoir à défendre le projet qu'en amont, au moment de l'audition face au jury. Je pensais qu'une fois ma candidature retenue, une fois le projet validé, ma présence ne serait pas remise en cause, questionnée par ceux-là même qui m'avaient sélectionnée. Pourtant, tout pouvait poser un problème : la musique, le corps, le geste, le sens du geste, l'éventualité d'un jeu, d'une dimension ludique ou, au contraire, la possibilité d'une dimension trop conceptuelle, jugée trop peu accessible à une majeure partie du public. Ce qui me semblait faire partie d'un tout contenu dans l'idée même de faire entrer «les arts du spectacle vivant » au musée devenait effrayant, dangereux, menaçant. Je n'ai jamais tout à fait compris sur qui ou quoi planait cette menace.

Est-ce seulement besoin de le préciser de nouveau ? Les enjeux qui étaient et sont toujours les miens - de recherche, de poésie, d'expérience et de partage, mais aussi de constat, d'analyse, de témoignage et parfois de critique - ne sont pas tout à fait les mêmes que ceux que portaient, alors, mes interlocuteurs. Nombreux, pourtant, ces interlocuteurs n'exprimaient pas tous non plus les mêmes attentes vis-à-vis de ce projet. Si le musée est vide, alors il faut le remplir par tous les moyens, disaient les uns. Les risques sont trop grands pour les œuvres, répondaient les autres. 
Je me suis vu répéter, souvent, que ce musée n'était pas le Louvre, qu'on ne pouvait pas y faire la même chose, que les espaces n'étaient pas adaptés, qu'ils étaient trop petits, trop vétustes, que le public n'était pas assez préparé à recevoir ce genre de proposition. Faut-il qu'il y ait des lieux non-dédiés plus dédiés que d'autres à la pratique du spectacle vivant ? Est-ce seulement possible? La performance elle-même doit-elle se restreindre aux lieux où elle serait a priori plus facilement présentable, à l'abri des scandales ou seulement des mécompréhensions d'un public surpris, bousculé par ce à quoi il ne s'attend pas ? Les grands musées ou les centres d'art contemporain doivent-ils seuls engager ce processus de lien avec le public, celui d'une médiation alternative ? Plus pragmatiquement, de quels moyens avonsnous besoin pour que le spectacle vivant et la recherche-création puissent avoir lieu ? De ma propre expérience, le peu d'espace disponible n'est qu'un frein partiel à une telle pratique. Il est une contrainte dont on peut aisément se jouer : proposer un mouvement minimal, une expérience de l'infra-regard, de l'infra-mouvement dont le spectateur averti (ou le créateur luimême, qu'il soit expérimenté ou néophyte) peut tout à fait se délecter, tant la sensation de se tenir dans le secret d'un dispositif dont on est le gardien est délicieuse. Le spectateur-acteur jouit de sa posture, de son éventuel décalage vis-à-vis de la norme admise et de la confiance ragaillardie que lui confère sa légitimité dans la mise en pratique du protocole performatif (convenu avec le lieu qui l'accueille et le valide, donc).

C'est bien sur ces points qu'il s'agit de se montrer précis. La question de la « performance » (cela qui, précisément, n'est ni le théâtre, ni la danse, mais qu'il convient encore de définir) appartient au champ des arts plastiques, elle témoigne de l'expansion même, dans le courant du vingtième siècle, des formes traditionnelles (ou conventionnelles) de la création artistique. Elle découle de l'intrusion duchampienne et post-duchampienne du geste dans l'acte de création et correspond à l'entrée en scène, dans l'espace muséal ou public, du corps, de la présence, du faire et surtout du vivant et donc de l'éphémère. Tous les musées peuvent-ils l'accueillir, s'en faire l'écrin ?

L'histoire de l'art du vingtième siècle a bien montré combien la performance se passe des écrins, des lieux. Elle n'a pas à être accueillie puisqu'elle est vivante, trouble les contours de la nature même de l'œuvre d'art et s'infiltre souvent là où elle n'est pas attendue. La performance a changé, pourtant, elle s'est institutionnalisée, presque domestiquée. Désormais, elle se cantonne souvent aux festivals bien dédiés et aux lieux qui proposent déjà une programmation contemporaine; au risque sans doute d'un entre-soi qui appauvrit les propositions et exclut, de fait, la participation d'un public plus éloigné de cette culture officielle ou considérée comme élitiste. En témoignent quelques récents exemples, pourtant de très 
grande qualité. En 2017, lorsque la Ferme du Buisson ${ }^{3}$ programme son évènement « Le Musée performé » dans le cadre de la seconde édition de son Performance Day (en lien avec le quarantième anniversaire du Centre Pompidou), elle annonce déjà :

Depuis les années 60-70, les artistes interrogent l'institution muséale, ses codes et ses discours. Comment constitue-t-elle ses collections ? Quelles sont les modalités d'exposition et de circulation des œuvres ? Quelle place attribue-telle aux spectateurs et à la transmission ? Aujourd'hui, toute une génération d'artistes renouvelle ces questionnements à travers des récits et des relectures alternatives ${ }^{4}$.

La question qui se pose est bien celle-là : de quoi parle-t-on, vraiment ? D'où parle-ton ? depuis quel champ disciplinaire ? depuis quel lieu et depuis quelles pratiques ? depuis quelles données préalables ? Qu'est-ce qui, dans cette démarche, doit être considéré comme nouveau, singulier, original ? Passionnante, la programmation du second Performance Day de la Ferme du Buisson tient pourtant lieu dans un espace qui, s'il interroge le muséal, ne s'y confronte pas directement. La performance n'y a tout simplement pas lieu. Toutefois, la cérémonie de Beatrice Balcou ${ }^{5}$, la conférence-performance de Kapwani Kiwanga $A$ Conservator's Tale ${ }^{6}$, et la proposition poétique de Ben Evans \& Luís Miguel Félix, An Invisible Museum $^{7}$, présentées toutes trois à cette occasion, demeurent à mes yeux des expériences et des inspirations capitales qui ont participé à construire ma démarche.

L'usage du terme de "performance», donc, dans ce contexte, doit être réévalué. Monstration anglosaxonne, forme courte, dispositif théâtral présenté dans un lieu non-dédié : qu'est-ce à dire ? Légeret, par exemple, ne souhaite pas utiliser le syntagme «spectacle vivant » pour désigner les actions de médiations alternatives et de recherches-créations qu'elle mène avec son équipe et ses étudiants depuis 2012. Je la rejoins sur ce point, lorsqu'elle explique : «Le terme performance est le plus approprié par l'importance du jeu éphémère et fictionnel dans les changements de rôle (acteurs et visiteurs) et la puissance d'effacement par les gestes des frontières entre catégories sociales et disciplinaires » (11). Les scènes de ces performances-là sont fictionnelles puisque, comme l'explique encore Légeret, elles n'ont pas d'espace balisé par l'institution. Elles ont lieu « en relation » aux œuvres exposées et au cœur d'un dispositif dans lequel les rôles peuvent être inversés. Souvent performées sous le mode de l'improvisation, elles créent toujours une surprise pour le grand public qui en est le spectateur tout à la fois privilégié et s'en trouve involontairement intégré. Décrivant l'éventualité d'une contagion des gestes, et d'une circulation des regards et des pratiques, même tacite, Légeret décrit ce qu'elle appelle la performance d' «être-ensemble dans l'espace muséal » et l'oppose à une autre perspective, celle défendue par Shannon Jackson, spécialiste des Performance 
Studies de l'université de Californie à Berkeley. La chercheuse américaine, presque à l'inverse, décrit et analyse l'émergence d'une nouvelle esthétique née de ces pratiques désormais récurrentes de constructions d'expériences éphémères dans l'espace muséal actuel. Le régime expérientiel et participatif serait selon elle conditionné, au service de l'économie néo-libérale : «En effet, l'esprit de la performance, semble être à la fois le symptôme et une réponse perspicace à un monde globalisé. [...] le paysage performatif du musée ou de la ville semble occuper les mêmes plans que les mondes du travail post-fordistes qui se conçoivent comme des expériences et des rencontres commerciales » (Beaufils et de Morant 79-85 et Légeret 10-11). Cette performance-là n'est pas celle que j'ai souhaité pratiquer au cours de ce projet. Ce n'est pas non plus celle que je défends aujourd'hui, avec le recul qui est le mien bientôt deux ans après que ce projet a eu lieu. Cette perspective, pourtant, explique en partie la raison pour laquelle je suis tant attachée à la question du théâtre - même à un théâtre hors les murs, détourné, trouvé ailleurs. Mon projet dans ce musée municipal d'art et d'histoire ne reposait pas exclusivement sur la question de l'expérience, du partage, ou même de la médiation alternative - bien que toutes ces facettes du projet aient été traversées elles aussi, dans un sens très semblable à celui que décrit Légeret. Mon projet reposait sur une approche que j'ai peu exposée à l'époque parce qu'elle constituait alors et constitue encore aujourd'hui une pensée en gestation. Je pensais pouvoir rematérialiser le rapport aux choses, aux objets, aux œuvres exposées, qu'elles soient patrimoniales ou issues de la collection des Beaux-Arts du musée. Je pensais inviter à prendre le temps et à regarder autrement, je pensais que la performance - ou le théâtre - pouvait permettre de retrouver le sens et la sensation de la découverte d'une œuvre d'art exposée, de la rencontre avec une œuvre d'art mise en présence. Je reste profondément attachée à la question du théâtre, de celle d'une théâtralité intrinsèque aux objets exposés, un parti-pris nourri par ma formation de marionnettiste, mais aussi par ma recherche doctorale et les lectures qui l'ont accompagnée. L'une d'entre elles, tout particulièrement, s'est révélée centrale.

\section{Rematérialiser la rencontre : la marionnette au centre du projet}

Dans son célèbre article de jeunesse «Art and Objecthood», le critique étatsunien Michael Fried explique que lorsqu'elle se trouve mise en scène et qu'elle semble imposer sa présence à un public, l'œuvre littéraliste (ou Minimaliste comme celle de Tony Smith, Frank Stella, Donald Judd ou Robert Morris, par exemple), en raison de son objectité, prend le spectateur au piège d'une situation de regard qui, pour lui, relève du théâtre, et donc de l'expérience, et annonce ainsi la fin de l'art. Ces concepts, tels qu'ils sont développés par Fried 
dans sa série d'ouvrages sur l'esthétique et l'origine de la peinture moderne ${ }^{8}$, s'intéressent donc exclusivement à ce qui n’appartient pas au champ du théâtre.

\section{Faire émerger le théâtre au musée}

Dans le langage de Fried, l' «Objectitié » (objecthood) correspond à ce que Clement Greenberg appelait déjà la « condition du non-art ${ }^{9} »$ (cité dans Fried 118), à propos du travail d'Anne Truitt qui, selon lui, anticipait le courant minimaliste. Cette condition, Greenberg la pensait dans la tridimensionnalité, cette dimension que se partagent à la fois la sculpture et tout objet matériel qui n'appartient pas au champ de l'art - une collection patrimoniale, par exemple.

Selon Fried, le littéralisme adhère à l'objectité comme s'il s'agissait d'une forme d'art à part entière, alors même que le modernisme se donne pour objectif d'invalider, à travers la forme, sa propre objectité. Les exigences de l'art, selon lui, sont aux antipodes des conditions de l'objectité, alors qu'elle est «hypostasiée », explique-t-il, par les littéralistes. Pourquoi ? Malgré des divergences que l'auteur choisit de laisser de côté ${ }^{10}$, Fried associe les œuvres de Morris et Judd mais aussi celles de Smith ou Stella en fonction d'un point commun fondamental : le refus de toute composition. Dans leur travail, la forme est l'objet. Tout ce qui se rapporte à la composition créerait un tout artificiel et relèverait ainsi d'une fallacieuse possibilité d'anthropomorphisme. Pour Judd, en effet, « l'aspect 'relationnel' qui [caractérise] la plupart des sculptures [relève] de l'anthropomorphisme»(Fried 116). Si la sculpture moderniste (contrairement au specific object, donc), est articulée et porte la marque du pluriel, la chose minimale, en revanche, se doit d'être unique et indivisible: une complétude à appréhender dans sa globalité. Selon lui, l'œuvre n'est pas relationnelle parce qu'elle est un tout (Gestalt) et tient ainsi le spectateur à distance, à la fois physiquement et psychiquement. Le spectateur est fait « sujet et l'œuvre... un objet [...] c'est l'objet, et non le spectateur, qui doit être au centre de la situation et en constituer le point de mire ; mais la situation elle-même appartient au spectateur - c'est sa propre situation » (121, il souligne). L'unicité de la forme garantit l'intégrité de l'objet, or, selon Fried, «c'est cette importance accordée à la forme qui explique l'impression, maintes fois exprimée par la critique, qu'un vide émane des sculptures de Judd et Morris » (117).

C'est autour de l'idée d'un tel «vide» qu'un premier problème est posé dans la réflexion menée par Fried. Il explique en effet que cette impression de vide qui émane de la plupart des œuvres littéralistes suppose l'existence d'un intérieur et par-là-même relève, de manière flagrante, de cet anthropomorphisme que Judd rejette pourtant ardemment : « tout se 
passe comme si l'œuvre avait une vie intérieure - voire une vie secrète » (124). L'auteur dénonce alors ce qui lui apparait comme l'une des principales aberrations du littéralisme et avance ainsi qu' «au cœur de la pratique et de la théorie littéralistes, [il y a] une sorte de naturalisme latent ou caché - un anthropomorphisme » (125). Pour argumenter son propos, il fait appel à Smith qui, très explicitement, déclare à propos de ses œuvres qu'il ne pense jamais à elles « comme à des sculptures mais comme à une certaine forme de présence ${ }^{11}$ » (cité dans Fried 125). L'exemple le plus célèbre parmi les sculptures-présences de Smith est sans doute Die (1962), un impressionnant cube aux dimensions étrangement humaines d'1m 83 de côté, et dont le mystère se présente sous la forme d'un célèbre jeu de questions-réponses :

$\mathrm{Q}$ : Pourquoi ne l'avez-vous pas fait plus grand, pour qu'il ait l'air de dominer le spectateur?

$\mathrm{R}$ : Je ne faisais pas un monument.

$\mathrm{Q}$ : Mais si vous l'aviez fait plus petit, le spectateur aurait pu regarder pardessus?

$\mathrm{R}:$ Je ne faisais pas un objet. (Smith, cité dans Fried 123)

Fried s'interroge au sujet du processus de création de l'œuvre de Smith, et surtout au sujet de son résultat : «Qu'a-t-il fait sinon un substitut de personne - une sorte de statue ?» (123). Comment, alors, la percevoir ? C'est la question que pose à son tour Georges DidiHuberman, en 1992, lorsqu'il fait de l'énigme de Die l'un des pivots de son ouvrage Ce que nous voyons, ce qui nous regarde, dont l'incipit est bien connu : «Ce que nous voyons ne vaut - ne vit - que par ce qui nous regarde. Inéluctable est pourtant la scission qui sépare en nous ce que nous voyons de ce qui nous regarde»(9). Comme en dialogue avec la critique américaine, Didi-Huberman propose de déceler comment la pratique spectatorielle peut s'extraire d'un clivage stérile entre la croyance et la tautologie qui supposerait qu'il n'est possible de voir que de deux façons : ou bien l'on croit aveuglément ce que l'on n'a pas vu, ou bien on ne voit pas plus loin que ce qui est effectivement là. Il s'agit donc de repenser les pratiques du voir et du regarder, d'envisager de nouvelles postures qui articulent la critique formaliste et la pensée phénoménologique. Ainsi, à propos du Die de Smith, si l'on choisit de croire au titre annoncé, on peut voir un tombeau (prophétique, menaçant, ou tout simplement réel et contenant les restes d'un mort qui reposerait à l'intérieur) ou au contraire préférer le percevoir comme le cube qu'il est - What you see is what you see, disait Stella, autre éminente figure du mouvement minimaliste. Didi-Huberman est à la recherche d'un dépassement de ce clivage, en cherchant à comprendre la dialectique du volume et du vide, à travers l'analyse sensible de cette distance paradoxale par laquelle l'œuvre d'art nous tient à l'écart, comme en respect. 
«Die », dit l'œuvre de Smith, comme à l'impératif. Elle est une injonction qui, par sa simplicité crue mais violente, minimaliste, exige en effet d'être prise en compte et met à distance. Pour Didi-Huberman, l'œuvre de Smith est un autoportrait. Si elle ne lui ressemble pas, elle l'imite cependant en fonction d'un critère essentiel, celui de ses dimensions, lui qui mesure effectivement $1 \mathrm{~m}$ 83. Bloc d'acier, elle n'en demeure pas moins un monolithe à taille humaine, presque une pierre tombale. Paradoxalement, l'œuvre se fait à la fois pure abstraction et portrait anthropomorphique qui (me) regarde. Selon Fried, le rapport sujet-objet opéré par la mise à distance de l'œuvre littéraliste «n'est pas sans présenter quelque ressemblance avec la mise à distance, ou l'envahissement, que représente la présence silencieuse d'une autre personne » (123). Or, si l'œuvre me regarde, alors elle n'est plus seulement objet, elle est devenue sujet de l'acte de regard : œuvre en acte, œuvre actrice, résolument animée. Si je ne la vois plus seulement mais la regarde et que ce regard m'est rendu, c'est un dialogue qui s'instaure non plus entre un sujet et un objet mais bien entre un sujet et un objet devenu à son tour sujet - même de façon éphémère.

\section{Les habitants du seuil ${ }^{12}$}

Ce sont bien là les présupposés théoriques que j'avais en tête au moment d'entrer au musée et d'y déployer mon projet. La radicalité de ma proposition - que je sous-estimais grandement au début de ce travail - reposait bien sur cette hypothèse : celle d'une théâtralité inhérente aux objets muséaux, et pas seulement littéralistes. Cette qualité de présence propre aux objets muséaux par lesquels une rencontre est possible, recèle à mes yeux une dimension marionnettique. Le regard seul du regardeur donne vie à ce qui est regardé. L'inquiétude et le trouble de cette présence silencieuse décrite par Fried sont devenus dans ma pratique artistique les mouvements d'une réanimation possible de l'objet exposé, cette personne possible en présence silencieuse.

Cette idée est donc également à l'origine de l'un des projets phares menés au cours de cette résidence, Les habitants $d u$ seuil, diptyque réalisé et présenté pour la première fois au cours de cette résidence. Le diptyque, comme deux rituels complémentaires, s'organise autour de mystérieux lièvres de tissu rapiécé. Un film retrace l'animation de ces objets fétiches en relation aux œuvres ensommeillées du musée ; tandis qu'une performance, appelée «pièce vivante » réactive l'expérience en invitant au dialogue entre un public et un espace muséal à (ré)animer.

Les « lagomorphes », du nom de cette famille à laquelle appartiennent les lièvres et les lapins, sont associés à l'idéal de l'abondance. Rapides, énergiques, à l'appétit sexuel débordant, 
ils dévorent et consomment tout ce qui les entoure. Ceux-là sont immobiles, placides, inanimés. Ils font écho à celui à l'oreille duquel murmure Joseph Beuys lors de sa performance Comment expliquer des tableaux à un lièvre mort? présentée à Düsseldorf en 1965. Beuys y engage avec l'animal inerte une communication non intellectuelle. Il rappelle que certaines cultures chamaniques, comme celles des Tatars de Crimée, considèrent les lièvres comme des animaux magiques et mystérieux. Creusant les galeries dans lesquelles ils se terrent, ils accèdent aux secrets de l'en-deçà du sol. Ce sont des animaux de passage, de transition, de frontière entre vie et non vie.

À l'inverse de leur image de compulsion et de l'exigence contemporaine de l'excès de consommation, ceux-là invitent plutôt à la contemplation, à un au-delà du temps. L'idée était de nouveau de combattre l'image d'un musée comme espace du passé et de l'archive inanimée. Il s'agissait, comme dans les arts de la marionnette, de ne pas se fier à l'inertie de la matière, de découvrir comment l'œuvre exposée peut encore vibrer. Ce projet a été celui partagé au début de ma résidence avec une cinquantaine d'enfants âgés de six à huit ans. Tous ont rencontré les œuvres du musée à travers l'expérience de l'animation d'une marionnette. À la manière de la performance de Joseph Beuys, les jeunes participants se sont vus en situation d'interaction et de transmission avec un objet inanimé, un impressionnant lièvre de tissu rapiécé. Ce sont alors autant les œuvres que l'objet marionnette auxquelles il leur faudra donner vie. Le film Les habitant du seuil est réalisé à partir de cette expérience ${ }^{13}$.

Ce film est un objet hybride qui continue de me passionner - bien qu'il soit évidemment imparfait - parce qu'il traverse l'ensemble des enjeux de ce projet, il recèle les éléments les plus fondamentaux qui ont construit l'ensemble de cette résidence. Il peut être perçu comme le document qui relate l'expérience vécue d'une rencontre entre deux classes d'enfants de banlieue parisienne avec leur musée municipal : comme une appropriation du territoire à partir de son patrimoine. C'est en effet une partie de son propos. Il expose aussi une première recherche, de trouver des moyens de création avec des enfants de si jeune âge, dont je n'avais aucune expérience. Comment les faire entrer au musée, comprendre le musée ? Comment, aussi, ne pas verser dans une forme de démagogie, de ne pas céder à la facilité, leur faire comprendre que tout n'est pas «joli », « agréable », « intéressant » ou « fragile »? Je me suis efforcée de les autoriser à la prise de parole libre, délivrée des clichés, du ce-qu'il-faut-dire pour faire plaisir ou plaire aux adultes. Comment leur faire respecter les règles d'un espace muséal (la patience, le calme, l'impossibilité de toucher les œuvres directement) tout en leur faisant comprendre que tout cela est un jeu dans lequel ils peuvent se fondre ? Après une visite commentée, ils étaient détenteurs, à leur manière, d'un savoir à eux, qu'ils pouvaient 
transmettre. La marionnette pouvait alors jouer ce rôle moteur qu'elle tient dans le film. Elle fait parler. Elle est un prétexte à une forme de remédiation de l'œuvre d'art, de leur réanimation par la prise en compte, le regard, la fable qui s'en raconte et la poésie qui en émerge : celle d'une traversée de multiples seuils.

De nombreux spectateurs et de participants m'ont demandé de leur donner le sens de ce titre, de leur expliquer de quel seuil il était question. Beaucoup détenaient pourtant déjà les réponses : le seuil de l'animé et de l'inanimé, de l'enfance et de l'âge adulte, du dedans et du dehors, de la connaissance et de l'ignorance. Pourtant, chacune des figures du film - enfant ou marionnette - est habitante de ce seuil, ne l'a pas totalement franchi. Elle construit son état de présence dans un va-et-vient permanent entre l'un et l'autre, dans une zone trouble et indéterminée, une zone d'indiscernabilité.

C'est cela que j'ai aussi souhaité développer dans le second volet de ce diptyque, en solo. Cette performance appelée «pièce vivante » se présente comme le revers du film. Au grand nombre d'enfants se substitue l'isolement de la marionnettiste que j'incarne, débordée par le nombre des marionnettes. Le rapport d'échelle s'est inversé. Comme ils sont privés de mouvements, la perception que l'on peut se faire de ces animaux de feutre oscille entre jouet d'enfant et fétiche bricolé. Dans leur immobilité, ils font exemple. Ils questionnent notre mobilité. Renouant avec la dimension rituelle inhérente aux arts de la marionnette, Les habitants du seuil s'est d'abord voulue comme une célébration de la contemplation muséale et comme une recherche sur la place de la marionnette au musée. Ont-elles droit, à leur tour, au statut d'œuvre d'art ? Leur entrée au musée en fait-elle des œuvres d'art ? Une à une, je les ai sorties d'une vieille malle délabrée, témoignage fictif d'une enfance révolue ou d'un passé dont on décide de raviver le souvenir. J'ai placé mes marionnettes chacune sur un socle, assise, stoïque, exposée-là comme une invitation à prendre le temps de regarder autour et de regarder chacune comme faisant partie du tout. À peine manipulés, Les habitants du seuil restent plutôt placides. Les lagomorphes que j'ai imaginés avec Nora Lesne, plasticienne factrice de marionnette et collaboratrice de longue date, n'en sont pas moins présents, et troublants. Leur regard peut percer celui du public qui, s'il s'attarde, pourra croire les voir bouger. C'est là que se joue leur drame et leur théâtralité.

Cette performance devait se jouer en musique et suivre une partition qui s'est peu à peu affinée au fil de la construction. Un élément en a été un déclencheur majeur. J'ai en effet démarré l'ensemble de la résidence avec un livre en poche : Le Terrier de Franz Kafka. Je ne l'avais jamais lu et petit à petit, page après page, et jour après jour, il me semblait que l'auteur était métaphoriquement en train de décrire ce que je vivais au musée. L'œuvre me faisait l'effet 
d'un miroir déformé de mon quotidien, de mes recherches, de mes errances. Un doute pourtant persistait, je ne savais pas qui j'étais censée représenter dans cette histoire : le narrateur ou l'envahisseur? Le texte de Kafka, on le connait, est un récit angoissé. Un personnage, dont personne ne connait l'identité, ni même la nature, déblatère sans discontinuer. Le récit à la première personne est celui d'un être fier de sa construction - Der Bau - que la traduction française à rendue animale : un terrier. Animal donc, dans sa version française, le personnage vit seul et se régale de la réussite de son ouvrage. Il est fier de ses galeries multiples, de leur sophistication, de leur fiabilité... mais quelque chose cloche. Il commence à entendre des bruits, perçoit des sons et des traces qui peu à peu l'obsèdent. Qu'elles soient réelles ou imaginaires, il ne peut plus détourner sa pensée de ces présences qui auraient pénétré son chezlui, son terrier, sa tanière, son refuge si bien organisé. Le chez-soi n'est plus sûr, il est menacé et devient menaçant. Qui est l'envahisseur de l'autre? Qui dérange celui qui s'était si bien installé ? L'ensemble de la proposition performative, qui se déroule pendant la lecture de ce texte, comme un parasite de la musique initialement imaginée, travaille cette ambiguïté. Qui est qui ? Dedans/dehors des galeries du musée, ou de l'accrochage permanent, qui était le parasite de quoi ?

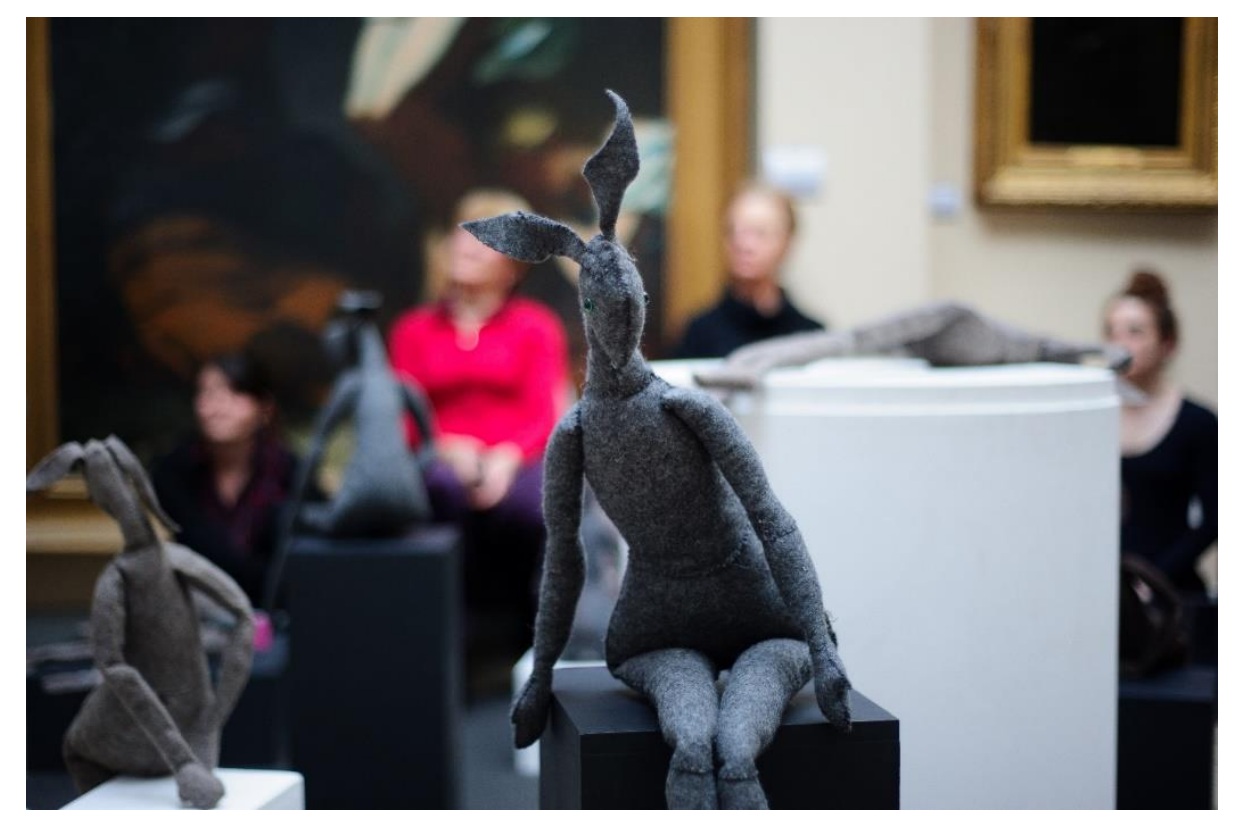

Fig. 2. Les habitants du seuil, pièce vivante d'après Joseph Beuys et Franz Kafka (Shirley Niclais, 2018)

\section{Bibliographie}

Agamben, Giorgio. Moyen sans fins, notes sur la politique (1990-1995). Paris : Rivages, 1995. Annycke, François « Un zeste d'artistique. Tentative de définition d'un geste artistique dans le cadre des CLEA, ARTS, ART2 ». $\underline{\text { www.en-toutes-lettres.eu }}$ 
Beaufils, Éliane et Alix de Morant, dir. Scènes en partage : l'être-ensemble dans les arts performatifs contemporains. Montpellier : Deuxième époque, domaine performance, 2018.

Chevalier, Pauline, Aurélie Mouton-Rezzouk et Daniel Urrutiaguer. Le musée par la scène : le spectacle vivant au musée : pratiques, publics, médiations. Montpellier : Deuxième époque, coll. À la croisée des arts, 2018.

Didi-Huberman, Georges. Ce que nous voyons, ce qui nous regarde. Paris : Minuit, coll. Critique, 1992.

Eidelman, Jacqueline, dir. «Mission Musées XXIème siècle - Inventez des musées pour demain », rapport de la commission « Musées XXIème siècle ». Ministère de la Culture et de la Communication, documentation française, 2017.

Foucault, Michel. «Des espaces autres (conférence de 1967)». Le corps utopique / Les hétérotopies. Paris : Lignes, 2009.

Fried, Michael. «Art et objectité [Art and Objecthood] » (1967). Tr. Fabienne Durand-Bogaert. Contre la théâtralité. Du minimalisme à la photographie contemporaine. Paris: Gallimard, coll. NRF essais, 2007.

Grenier, Catherine. La fin des musées ? Paris : Regard, 2013.

Légeret, Katia, dir. Créons au musée. Performances des arts vivants. Paris : Geuthner, 2019.

Niclais, Shirley. « Ci-gît la marionnette. Réifications et réanimations de l'humain sur les scènes de Tadeusz Kantor et de Louise Bourgeois », thèse de doctorat sous la direction d'Evelyne Grossman, Sorbonne Paris Cité, Université Paris Diderot, laboratoire CERILAC, 2019.

${ }^{2}$ Docteure en philosophie, Katia Légeret est chercheure et professeure des universités en esthétique des arts de la scène à l'université Paris 8 et directrice de l'équipe de recherche EA 1573 « Scènes du monde, création, savoirs critiques ». Elle est metteure en scène, chorégraphe, artiste de bharata-nāțyam sous le nom de Manochhaya et spécialiste des théâtres dansés de l'Inde.

${ }^{3}$ Le Ferme du Buisson porte un projet résolument transdisciplinaire en ce qu'il regroupe, au sein d'un même lieu, un cinéma, un centre d'art contemporain et une scène nationale.

${ }^{4}$ Un événement en lien avec la programmation du quarantième anniversaire du Centre Pompidou, en collaboration avec le Centre Photographique d'Île-de-France, le FRAC Ile-de-France et Serralves - Museu de Arte Contemporânea (Porto), leurs dirigeants en sont les co-commissaires et programmateurs : Julie Pellegrin (directrice Centre d'art contemporain de la Ferme du Buisson), Xavier Franceschi (directeur du FRAC Ile-deFrance), Nathalie Giraudeau (directrice du Centre photographique d'Ile-de-France) et, à la Fundação de Serralves - Museu de Arte Contemporânea, Cristina Grande (programmatrice danse et performance), Ricardo Nicolau (commissaire d'exposition), Pedro Rocha (programmateur musique).

https://www.lafermedubuisson.com/programme/performance-day-2017

${ }^{5}$ Les Cérémonies sans titre de Béatrice Balcou « sont des performances réalisées avec une œuvre d'art dont elle n'est pas l'auteur, choisie préalablement dans une collection publique ou privée. Toutes les cérémonies développent une temporalité particulière autour d'un évènement : celui de la mise en exposition de l'œuvre.
} 
À travers ses gestes précis et délicats, Balcou s'intéresse à l'attention portée à la matérialité de l'œuvre d'art autant que sur le comportement de celui qui la regarde. Ici, l'œuvre n'est pas une image éphémère à reconnaître rapidement ou à consommer, mais une matérialité physique dont il faut prendre soin, et avec laquelle passer du temps collectivement ».

https://www.lafermedubuisson.com/programme/centre-dart/archives_centre-d-art2016-2017/beatrice-balcou

${ }^{6}$ A Conservator's Tale est une conférence-performance présentée par l'artiste Kapwani Kiwanga qui, « Nourrie d'une formation en sciences sociales, [...] crée des protocoles qui agissent comme des filtres à travers lesquels elle observe les cultures et leurs capacités de mutation. Elle y met volontairement à mal la frontière entre vérité et fiction afin de perturber les récits hégémoniques. Dans ses conférences-performances, elle construit des récits scientifiques nourris d'afrofuturisme, de science-fiction, de fables populaires et de documents intangibles afin de confronter archives et figures invisibles. Elle endosse ici le rôle d'une conservatrice-archiviste pour faire parler des objets muets, manipuler des objets invisibles et relater des récits oubliés, où faits historiques ignorés, anecdotes insolites et bêtes mythiques ont leur place. En mêlant lecture, son et extraits vidéo, l'artiste questionne le statut du document, du classement et les potentialités de la transmission orale pour projeter un musée immatériel ». https://www.lafermedubuisson.com/programme/kapwani-kiwanga-a-conservators-tale

7 The Invisible Museum s'installe pendant une semaine au Centre d'art dans l'exposition en cours: «SoixanteDixSept : Quand Rossellini filmait Beaubourg ». Un musée dans un musée. L'un vient parasiter l'autre. Mais il n'y a rien à voir dans The Invisible Museum. Sa collection consiste en descriptions d'objets, d'expériences et de situation «offertes » par les visiteurs aux médiateurs. Les œuvres exposées dans ce musée invisible se matérialisent au travers de visites guidées proposées par des médiateurs qui les décrivent de mémoire. Sa collection évolue, change d'aspect, voire disparaît, selon les souvenirs de chaque guide et des artistes. À travers cette intervention, Ben Evans et Luis Miguel Félix questionnent la transmission des œuvres et la définition même du musée. Comment le musée devient-il une entité concrète ? Où et comment ses collections se matérialisentelles ? Dans quelle économie s'inscrit-il ? https://www.lafermedubuisson.com/programme/ben-evans-et-luismiguel-felix

${ }^{8}$ Voir aussi Michael Fried, La place du spectateur. Esthétique et origine de la peinture moderne 1. [Absorption and Theatricality. Painting and Beholder in the Age of Diderot, 1980], tr. de l'anglais Claire Brunet. Les volumes 2 et 3 sont respectivement consacrés au Réalisme de Courbet (publié en français en 1993) et au Modernisme de Manet (publié en français en 2000).

${ }^{9}$ Voir aussi le recueil Art et culture, essais critiques (1961), tr. de l'anglais Ann Hindry.

${ }^{10}$ Le travail de Donald Judd est d'abord soutenu par un refus de l'illusion picturale. La peinture s'épuise et ne peut proposer qu'un nombre limité de réponses au problème qu'elle pose fondamentalement : l'organisation de la surface plane picturale. Il faut donc, pour Judd, privilégier la tridimensionnalité mais il ne considère pas pour autant ses objets spécifiques comme des sculptures. En revanche, Robert Morris situe son travail dans le prolongement d'un constructivisme russe, porté, par exemple, par Alexandre Rodtchenko ou Naum Gabo. Fried évacue le problème par cette simple phrase : «ces divergences, cependant, sont moins importantes que ce qui fait la communauté de vues entre Judd et Morris » (15).

${ }^{11}$ Je reconnais, cependant, que le raccourci qu'il opère entre le travail de Donald Judd, érigé en unique théoricien, et la sensibilité de Tony Smith est assez réducteur, voire hasardeux.

${ }^{12}$ Les habitants $d u$ seuil, diptyque film et pièce vivante, de deux fois 20 minutes. Premières étapes les 9 et 10 mars 2018 dans le cadre du Printemps des poètes, et 21 mars à l'Université de Paris Nanterre, dans le cadre du Festival MARTO.

${ }^{13}$ Ce film peut être visionné en ligne, à cette adresse : https://vimeo.com/265803240 (mot de passe : lagomorphes) 